\title{
Structural basis for a homodimeric ATPase subunit of an ECF transporter
}

\author{
Chengliang Chai ${ }^{1,3^{*}}$, You $\mathrm{Yu}^{2^{*}}$, Wei Zhuo ${ }^{2^{*}}$, Haifeng Zhao ${ }^{2}$, Xiaolu $\mathrm{Li}^{2}$, Na Wang ${ }^{2}$, Jijie Chai ${ }^{1,2}$, \\ Maojun Yang ${ }^{2 \bowtie}$ \\ ${ }^{1}$ College of Biological Sciences, China Agricultural University, Beijing 100083, China \\ ${ }^{2}$ MOE Key Laboratory of Protein Sciences, Tsinghua-Peking Center for Life Sciences, School of Life Sciences, Tsinghua \\ University, Beijing 100084, China \\ ${ }^{3}$ National Institute of Biological Sciences, Beijing 102206, China \\ $\triangle$ Correspondence: maojunyang@tsinghua.edu.cn
}

Received August 9, 2013 Accepted September 9, 2013

\begin{abstract}
The transition metal cobalt, an essential cofactor for many enzymes in prokaryotes, is taken up by several specific transport systems. The CbiMNQO protein complex belongs to type-1 energy-coupling factor (ECF) transporters and is a widespread group of microbial cobalt transporters. CbiO is the ATPase subunit (A-component) of the cobalt transporting system in the gram-negative thermophilic bacterium Thermoanaerobacter tengcongensis. Here we report the crystal structure of a nucleotide-free $\mathrm{CbiO}$ at a resolution of 2.3 $\mathrm{A}$. CbiO contains an $\mathrm{N}$-terminal canonical nucleotide-binding domain (NBD) and C-terminal helical domain. Structural and biochemical data show that CbiO forms a homodimer mediated by the NBD and the C-terminal domain. Interactions mainly via conserved hydrophobic amino acids between the two C-terminal domains result in formation of a four-helix bundle. Structural comparison with other ECF transporters suggests that non-conserved residues outside the T-component binding groove in the A component likely act as a specificity determinant for T components. Together, our data provide information on understanding of the structural organization and interaction of the CbiMNQO system.
\end{abstract}

KEYWORDS CbiO, Cobalt, ECF, ATPase, Thermoanaerobacter tengcongensis

\section{INTRODUCTION}

ATP-binding cassette $(A B C)$ transporters play critical roles in cells by translocating various substrates, ranging from small

*These authors contributed equally to the work.

(c) Higher Education Press and Springer-Verlag Berlin Heidelberg 2013 ions to large macromolecules, across membranes (Dean and Allikmets, 1995). An intact ABC transporter consists of two variable hydrophobic transmembrane domains (TMDs) and two conserved hydrophilic ABC-ATPases, forming a translocation pathway energized by binding and hydrolysis of ATP (Dean et al., 2001). Energy-coupling factor (ECF) transporters are a new group of $A B C$ transporters in prokaryotes. Similar to other typical $A B C$ transporters, the new class of transporters contains two homologous or identical ATPases (A component), which associate with the transmembrane domains (T components), forming an ECF module (Zhang et al., 2010; Erkens et al., 2011). In contrast with the canonical $A B C$ transporters that employ soluble substrate binding proteins (SBPs), membrane proteins in ECF transporters is exclusively responsible for substrate binding (therefore termed S component) (Berntsson et al., 2012; Majsnerowska et al., 2013). An ECF module can be shared by highly diverse $S$ components, thus allowing the transport of chemically different substrates via the same complex (Fisher et al., 2012).

Cobalt $\left(\mathrm{Co}^{2+}\right)$ plays important roles in a variety of metabolic processes in prokaryotes by acting as a cofactor of enzymes, such as Vitamin $\mathrm{B}_{12}$ (Felsenstein, 1981; Mulrooney and Hausinger, 2003). In prokaryotes, the ABC-transporter systems and secondary permeases of the nickel/cobalt transporter (NiCoT) family are the two major types of high-affinity cobalt transporters (Eitinger et al., 2005). One of the ABC transporters, called the CbiMNQO protein complex, belongs to type-I energy-coupling factor (ECF) transporters and is a widespread group of microbial transporters for cobalt (Rodionov et al., 2006). CbiO from the CbiMNQO protein complex corresponds to an A component (Siche et al., 2010).

Recent structural studies of the ECF transporters with two 
homologous A components provide significant insight into the molecular mechanism underlying transportation of this novel family of ABC transporter (Karpowich and Wang, 2013; Wang et al., 2013). But whether and how the two identical A components in the ECF transporters interact with each other remain to be answered. Additionally, it is still not well understood how the conserved A components interact with different T components. In the present study, we reported the crystal structure of $\mathrm{CbiO}_{\text {TTE2260 }}$ at $2.3 \AA$ resolution. The structure reveals that $\mathrm{Cbi}-$ $\mathrm{O}_{\text {TTE2260 }}$ contains a typical N-terminal ATPase domain (Hung et al., 1998; Chen et al., 2003; Li et al., 2013) and a C-terminal helical domain. Structural and biochemical data demonstrate that $\mathrm{CbiO}_{\text {TTE2260 }}$ forms a homodimer. $\mathrm{CbiO}_{\text {TTE2260 }}$ dimerization is mainly mediated by its C-terminal two helices that form a four-helix bundle with those from a $2 \mathrm{nd} \mathrm{CbiO}_{\text {TTE2260 }}$ molecule. Structural comparison shows that Arg82 in $\mathrm{CbiO}_{\text {TTE2260 }}$ important for the interaction within an ECF module of other ECF transporters adopts a strikingly different rotamer. The different rotamer of Arg82 in $\mathrm{CbiO}_{\text {TTE2260 }}$ is caused by the non-conserved residues outside its T-component binding groove, suggesting that these non-conserved residues may be an epitope for interaction of A components with different $T$ units.

\section{RESULTS}

Structure determination and overall structure of CbiO $_{\text {TTE2260 }}$

Wild type $\mathrm{CbiO}_{\text {TTE2260 }}$ protein with a C-terminal $6 \times$ His tag was purified to homogeneity and crystallized in the absence of nucleotide and $\mathrm{Mg}^{2+}$ (Fig. 1A). The crystals of $\mathrm{CbiO}_{\text {TTE2260 }}$ belonged to space group $P 2{ }_{1} 2{ }_{1} 2$, with unit-cell parameters $a=$ 110.22 $\AA, b=115.16 \AA, c=54.42 \AA, \alpha=\beta=\gamma=90^{\circ}$ and two $\mathrm{CbiO}_{\text {TTE2260 }}$ molecules per asymmetric unit. The structure of $\mathrm{CbiO}_{\text {TTE2260 }}$ was solved using molecular replacement and further refined to a resolution of $2.3 \AA$ with $R_{\text {work }}=20.0 \%$ and $R_{\text {free }}$ $=25.1 \%$ (Table 1$)$. The finally refined structure contained residues 1-283 with excellent stereochemistry except Leu114 that was not well defined by electron density in both $\mathrm{CbiO}_{\text {TTE2260 }}$ molecules.

The monomeric structure of $\mathrm{CbiO}_{\text {TTE2260 }}$ can be divided into two domains: an N-terminal nucleotide-binding domain (NBD, residues 1-240) with canonical ATPase topology as observed in many other $\mathrm{ABC}$ transporters and a $\mathrm{C}$-terminal domain (residues 241-283) with two anti-parallel helices (Fig. 1B). In addition to a covalent linkage, the two domains of $\mathrm{CbiO}_{\text {TTE2260 }}$ also form hydrophobic and hydrogen bonding interactions (not shown). Interactions of the two domains result in formation of a triangle-shaped structure. The N-terminal NBD can be further divided into two subdomains, a RecA-like subdomain (residues 1-87 and 162-240) and a helical subdomain (residues $88-161)$. The former has an $\alpha / \beta$ folding motif with eight $\beta$-sheets and six $\alpha$-helices as found in the RecA-like subdomain of many other ATPases. Walk A (residues 40-48) and Walk B (residues 165-168) motifs conserved among all the ATPases and critical for nucleotide binding (Story et al., 1992) constitute the core of
Table 1. Statistics of data collection and refinement

\begin{tabular}{|c|c|}
\hline Parameters & Data set \\
\hline \multicolumn{2}{|l|}{ Data collection statistics } \\
\hline \multicolumn{2}{|l|}{ Cell parameters } \\
\hline$a(\AA)$ & 110.22 \\
\hline$b(\AA)$ & 115.16 \\
\hline$c(\AA)$ & 54.42 \\
\hline$\alpha, \beta, \gamma\left({ }^{\circ}\right)$ & $90,90,90$ \\
\hline Space group & $P 2{ }_{1}{ }_{1} 2$ \\
\hline Wavelength used $(\AA)$ & 0.9792 \\
\hline Resolution $(\AA)$ & $40.0-2.30(2.38-2.30)$ \\
\hline No. of all reflections & 124260 \\
\hline No. of unique reflections & 30742 \\
\hline Completeness (\%) & $97.9(99.5)$ \\
\hline Average $\mathrm{I} / \sigma(\mathrm{I})$ & $12.16(2.35)$ \\
\hline$R_{\text {merge }}(\%)$ & $13.3(68.5)$ \\
\hline Redundancy & $4.0(4.0)$ \\
\hline Wilson $B$ factor $\left(\AA^{2}\right)$ & 27.5 \\
\hline \multicolumn{2}{|l|}{ Refinement statistics } \\
\hline Resolution $(\AA)$ & 2.30 \\
\hline$R_{\text {work }}(\%)$ & 20.0 \\
\hline$R_{\text {free }}(\%)$ & 25.1 \\
\hline r.m.s.d. from ideal bond lengths $(\AA)$ & 0.005 \\
\hline r.m.s.d. from ideal bond angles $\left({ }^{\circ}\right)$ & 1.228 \\
\hline B-value $\left(\AA^{2}\right)$ & 36.72 \\
\hline \multicolumn{2}{|l|}{ No. atoms } \\
\hline ALL & 4728 \\
\hline Protein atoms & 4448 \\
\hline Solvent atoms & 195 \\
\hline Others & 85 \\
\hline \multicolumn{2}{|l|}{ Ramachandran plot } \\
\hline Res. in favored regions (\%) & 91.2 \\
\hline Res. in additional allowed regions (\%) & 8.4 \\
\hline Res. in disallowed regions (\%) & 0.4 \\
\hline
\end{tabular}

Values in parentheses are for the highest resolution shell. $R=\Sigma \mid F_{o b s}{ }^{-}$ $F_{\text {calc }} / \Sigma_{\text {Fobs }}$, where $F_{\text {calc }}$ is the calculated protein structure factor from the atomic model ( $R_{\text {free }}$ was calculated with $5 \%$ of the reflections).

the RecA-like subdomain. The helical subdomain contains four $\alpha$-helices ( $\alpha 3, \alpha 4, \alpha 5$ and $\alpha 6)$ and harbors the $A B C$ signature motif (residues 144-148, LSGGQ) that connects strand $\beta 4$ and strand $\beta 5$ in the RecA-like domain. (All structural figures in this paper were generated by PyMOL).

\section{$\mathrm{CbiO}_{\text {TTE2260 }}$ forms a C-terminal domain-mediated homodimer}

The nik, cbi, and bio gene cassettes encode a single A component and the proteins encoded by them presumably form a homodimer for a functional importer, although evidence for this 
A
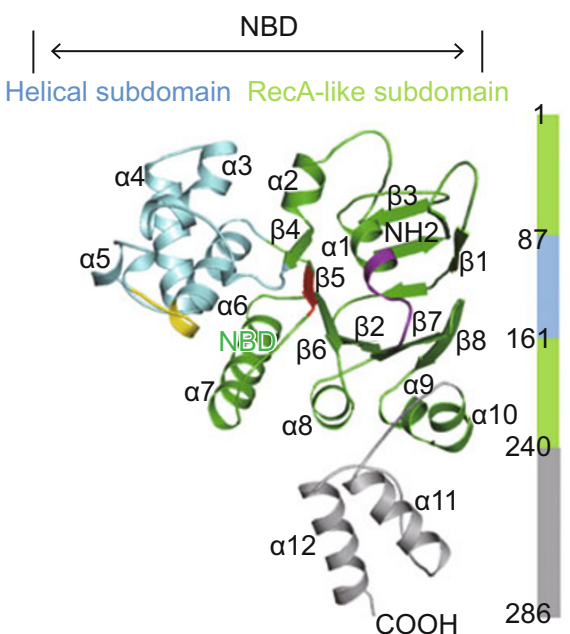

C-terminal helical domain
Figure 1. Sequence and structure of $\mathrm{CbiO}_{\text {TTE2260. }}$ (A) Subunit structure (molecule A in the asymmetric unit) with the nucleotide binding domain (NBD) (residues 1-240) in green/cyan color and C-terminal helical domain (CTD) (residues 241-286) in gray color. Different colors further distinguish the subdomains or segments in the NBD: green, RecA-like subdomain (residues 1-87 and 162-240); cyan, helical subdomain (residues 88-161); purple, Walker A motif; red, Walker B motif; yellow, ABC signature motif (LSGGQ). (B) The sequence of $\mathrm{CbiO}_{\text {TTE2260, }}$, secondary structure elements refer to $\mathrm{CbiO}_{\text {TTE2260 }}$ and stain the same color in A. Cylinder represents $\alpha$-helix, arrow represents $\beta$-strand and line represents loop. The residues of Walk $A$, Walk $B$ and $A B C$ signature motif were shown the same color in $(A)$.

B

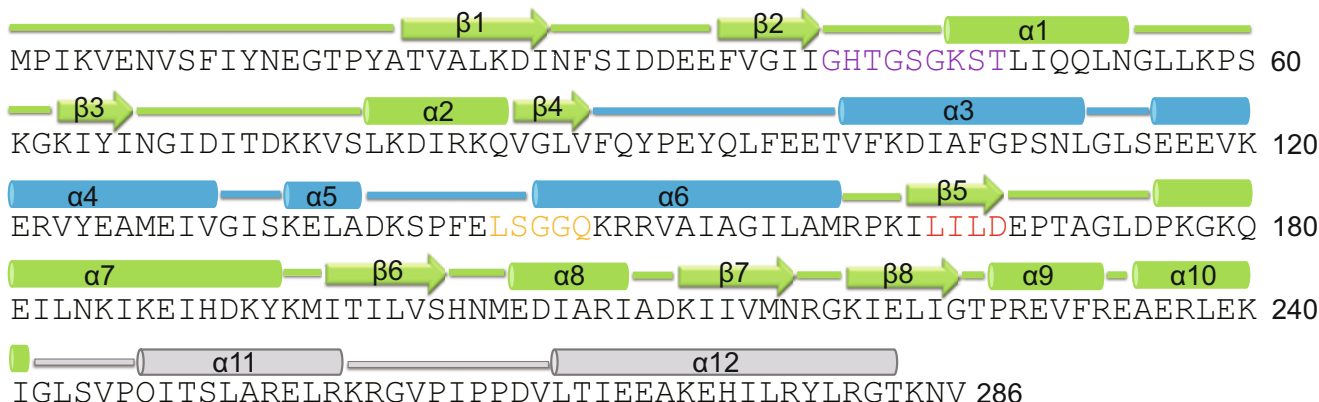

I GLSVPQITSLARELRKRGVPI P P DVLTIEEAKEHI LRYLRGTKNV 286

is lacking. Indeed, the two monomeric $\mathrm{CbiO}_{\text {TTE2260 }}$ molecules from one asymmetric unit form a homodimer (Fig. 2A) with a total burial of approximately $2860 \AA^{2}$ of surface area. Both of the two $\mathrm{CbiO}_{\text {TTE2260 }}$ domains are involved in the dimeric assembly, but the more extensive contacts stem from interactions of the two C-terminal helical domains. The two helices of this domain from one $\mathrm{CbiO}_{\text {TTE2260 }}$ molecule form a four-helix bundle with those of from the other one mainly via hydrophobic contacts (Fig. 2B). Many amino acids, in particular those (lle248, Leu251, Ala252, Leu255, Ile269, Ile276, Leu277 and Leu280) participating in the formation of the four-helix bundle, are highly conserved among the ECF type cobalt transporter members (Fig. 2C), suggesting that homodimerization is conserved mechanism of this family transporter. In addition to the hydrophobic contacts, polar interactions from periphery also contribute to the formation of the helical bundle. For example, Lys273 from one monomeric $\mathrm{CbiO}_{\text {TTE2260 }}$ makes a salt bridge with Glu254 from the other one (Fig. 2B). CbiO TTE2260 dimerization is also mediated by N-terminal RecA-like domain. Specifically, the $\mathrm{N}$-terminal end of $\alpha 7$ establishes contacts with a few loops from the other RecA-like domain (Fig. 2A). Our structural analyses strongly suggest that $\mathrm{CbiO}_{\text {TTE2260 }}$ is dimeric in crystals. To further support the structural observation, we quantified the molecular weight of $\mathrm{CbiO}_{\text {TTE2260 }}$ in solution using a gel filtration assay. Fully supporting the structural observation, the $\mathrm{CbiO}_{\text {TTE2260 }}$ protein was eluted at the position of $14.5 \mathrm{~mL}$, between those of the standard proteins with a molecular weight of $75 \mathrm{kDa}$ and $43 \mathrm{kDa}$ (Fig. 2D), indicating that $\mathrm{CbiO}_{\text {TTE2260 }}$ also formed a dimer in solution. Together, our data support the idea that the $\mathrm{CbiO}_{\text {TTE2260 }}$ protein forms a homodimer for further assembly of a functional transporter.

\section{Structural comparison with other $A B C$ transporters}

The DALI search for structural homologues of $\mathrm{CbiO}_{\text {TTE2260 }}$ identified many ATPases with a $Z$ score more than 10.0 . Among them, the A component of the quaternary complex structures of EcfSTAA' of Lactobacillus brevis (L. brevis) is the closest one with an r.m.s.d $1.5 \AA$ over 282 aligned $\mathrm{Ca}$ atoms (Fig. 3A). The structural organization of the dimeric $\mathrm{CbiO}_{\text {TTE2260 }}$ resembles that of the $A-A^{\prime}$ in ECF, while the latter is heterodimeric. More notably, the $C$-terminal domains of $A$ and $A^{\prime}$ from the ECF transporter also form a four-helix bundle, which can be well superimposed with that from the dimeric $\mathrm{CbiO}_{\text {TTE2260. }}$ The architecture of $\mathrm{N}$-terminal domain in the dimeric $\mathrm{CbiO}_{\text {TTE2260 }}$ is also similar to those of the $\mathrm{ABC}$ transporters (in semi-open conformation) MalK_E.c (Chen et al., 2003), and BtuD_E.c (Korkhov et al., 2012) with an r.m.s.d of $2.04 \AA$ over 373 aligned $\mathrm{C} \alpha$ atoms and $2.95 \AA$ over 219 aligned $\mathrm{C} \alpha$ atoms, re- 
A

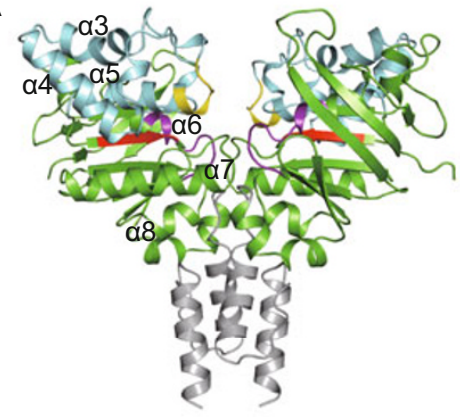

C

CbiO T.t 241 IGLSVPQITSIAREIRKRGVPIP CbiO_T.p 241 IGLGVPQITSIAREIQKKGVAIP CbiO C.a 242 FGLGVPQ TY TMEIKKKGKDIN CbiO_C.S 242 IGLGVPQVTYLAREIRKKGFNIS CbiO_A.m 242 IGLGVPQITYLMN_KNQGVHLR

CbiO_T.t PDVLTIEEAKEHILRY RGTKNV 286 CbiO T.p EDILTIEEAKEYIVRY RGTKNV 286

CbiO_C.a ENIFTVDEAKEEILKY RRKKDA 287

CbiO_C.S ENIFTIEEAKKELISILKVKH-- 285

CbiO_A.m DDILTVEEAKEEILKWIRSQNDA 287
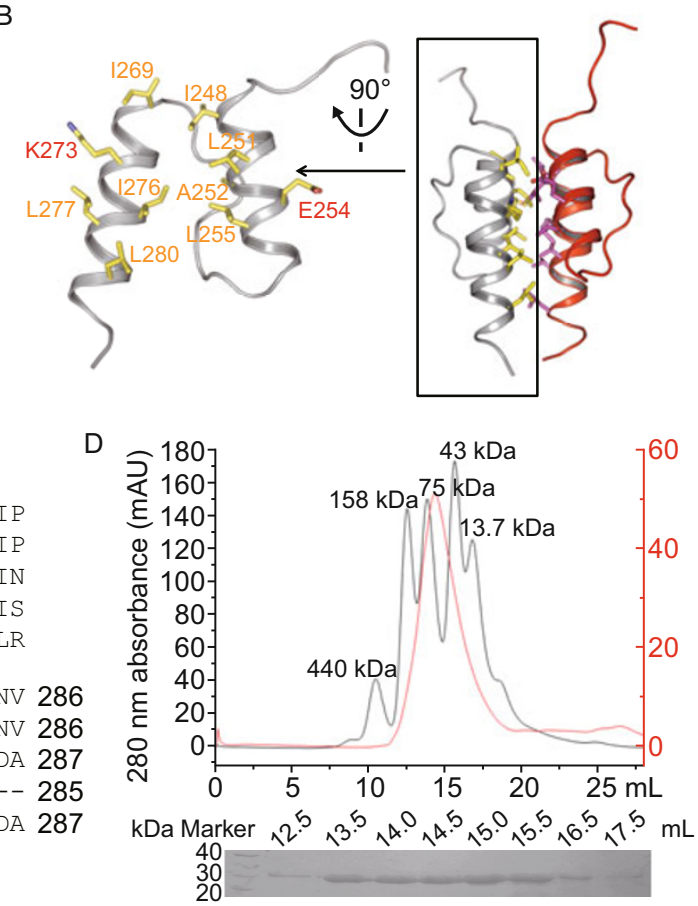

Figure 2. Homodimer of $\mathrm{CbiO}_{\text {TTE2260. }}$ (A) The homodimer consists of two $\mathrm{CbiO}_{\text {TTE2260 }}$ molecules and the color schemes are same to that shown in Fig. 1. (B) The four-helix bundle in a $\mathrm{CbiO}_{\text {TTE2260 }}$ homodimer. Side chains of the residues contributed to the formation of the helical bundle were shown as sticks. The residues colored dark yellow involve in hydrophobic contacts while colored red involve in salt bridge contacts. (C) Sequence alignment of C-terminal helical domains between CbiOTTE2260_T.t, CbiO_T.p, CbiO_C.a, CbiO_C.s and CbiO_A.m. Residues participated in the hydrophobic interaction are colored dark yellow and residues participated in the coulombic interaction are colored in red. (D) The profile of gel filtration calibration protein marker and $\mathrm{CbiO}_{\text {TTE2260 }}$ on a Superdex-200. Black and red curves represent protein marker and $\mathrm{CbiO}_{\text {TTE2260, }}$, respectively. X-axis is elution volume, black and red Y-axes are the $280 \mathrm{~nm}$ absorption of filtration calibration protein marker and $\mathrm{CbiO}_{\text {TTE2260 }}$, respectively. The SDS-PAGE of $\mathrm{CbiO}_{\text {TTE2260 }}$ is shown below.

spectively (Fig. 3B). Despite the conserved dimeric assembly, the structural elements mediating dimerization vary substantially among them. In all the structures of the dimeric $A B C$ transporters, the $\mathrm{N}$-terminal end of $\alpha 7$ is involved in their dimerization, though the detailed interactions are varied. However, these ABC transporters (Fig. 3C), such as MalK_E.c (Chen et al., 2003), BtuD_E.c (Korkhov et al., 2012), ClcV_S.s (Verdon et al., 2003), CysA_A.a (Scheffel et al., 2005) and DppD_T.t (Li et al., 2013), have non-conserved C-terminal domains that mediate their homodimerization.

\section{Arg82 from $\mathrm{CbiO}_{\text {TTE2260 }}$ exhibits a different rotamer from other ECF transporters}

The energy-coupling AT module interacts with different integral membrane proteins (S components) that dictate the substrate specificity of an active ECF transporter. The striking similarity between the dimeric $\mathrm{CbiO}_{\text {TTE2260 }}$ and the heterodimeric EcfAEcfA' prompted us to investigate why they assemble into different ECF modules and consequently different transporters for Co and vitamin transportation, respectively. Interaction of the T component with EcfA-EcfA' mainly via packing two conserved but not identical helices against the two similar $A$ components in the ECF transporter (Wang et al., 2013; Xu et al., 2013). The two conserved helices that contain the ARG (Ala-Arg-Gly) motif bind to a groove formed between the two subdomains of the NBD domain in each of the two A components. Unexpectedly, nearly all the T-component interacting residues are conserved (Fig. 4A) and similarly positioned (Fig. 4B) between EcfA or EcfA' and $\mathrm{CbiO}_{\text {TTE2260. The side }}$ chain of conserved Arg82 from $\mathrm{CbiO}_{\text {TTE2260, however, exhibits }}$ a markedly different rotamer from that of its equivalent Arg84 in EcfA or Arg80 in EcfA'. In $\mathrm{CbiO}_{\text {TTE2260, }}$ Arg82 is rotated to a position that completely overlaps with the ARG motif from the $T$ component of EcfA (Fig. 4B), suggesting that this residue may have a role in $\mathrm{CbiO}_{\text {TTE2260 }}$ selection against the $\mathrm{T}$ component in the Ecf transporter. In principle, the different rotamers of Arg observed in the ATPase domains can be induced by binding of $\mathrm{T}$ unit. However, this seems less likely, because Arg107 in the free EcfA (Karpowich and Wang, 2013) adopts a similar conformation to that of the T-component bound EcfA (Wang et al., 2013; Xu et al., 2013) (Fig. 4B). The positioning of Leu78 in $\mathrm{CbiO}_{\text {TTE2260 }}$ does not allow Arg112 to adopt a similar rotamer to that of Arg114 in EcfA. Additionally, Phe114 that interacts with the $T$ component via packing the main chain of the ARG motif 
A
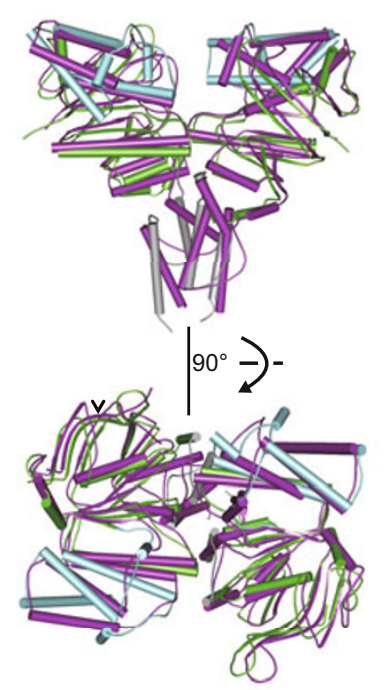

B
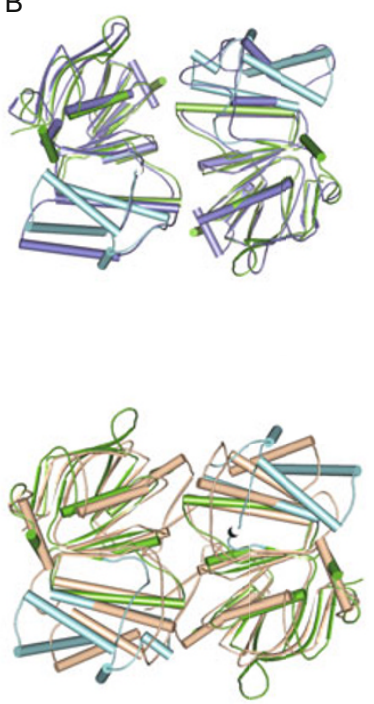

C

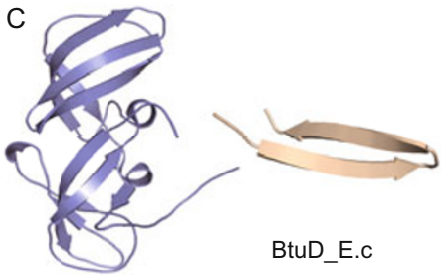

MalK_E.c

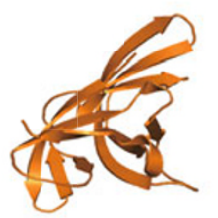

GIcV_S.S

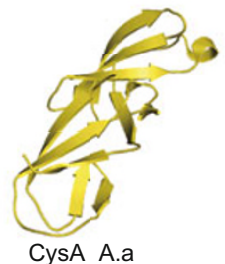

$\rightarrow 2$

DppD_T.t
CysA_A.a

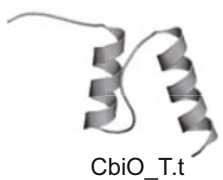

Figure 3. Structural alignment between CbiOTTE2260 and other ATP-binding subunits. (A) Structural alignment between $\mathrm{Cb}_{\text {TTE2260_T.t }}$ homodimer (same colored in Fig. 1) and EcfA-A'_L.b heterodimer (purple). (B) Structural alignment of NBDs between CbiO 1-240, green and cyan), MalK_E.c (residues 1-233, slate) and BtuD_E.c (residues 1-229, wheat). (C) Cartoon representation of C-terminal domains of MalK_E.c (residues 234-370, slate), BtuD_E.c (residues 231-249, wheat), GlcV_S.s (residues 243-352, orange), CysA_A.a (residues 246-355, yellow), DppD_T.t (residues 270-321, red) and CbiO TTE2260_T.t (residues 241-283, gray).

A

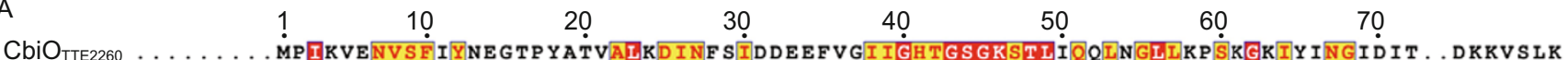

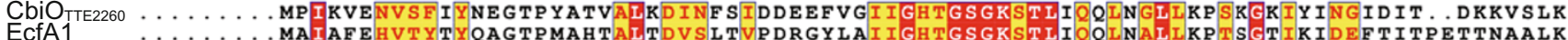
ECFA1
ECFA2

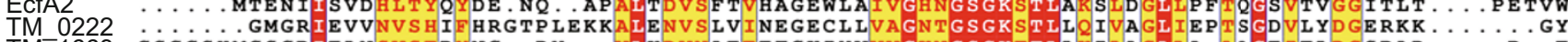

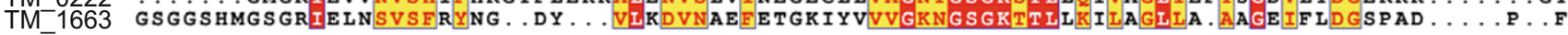

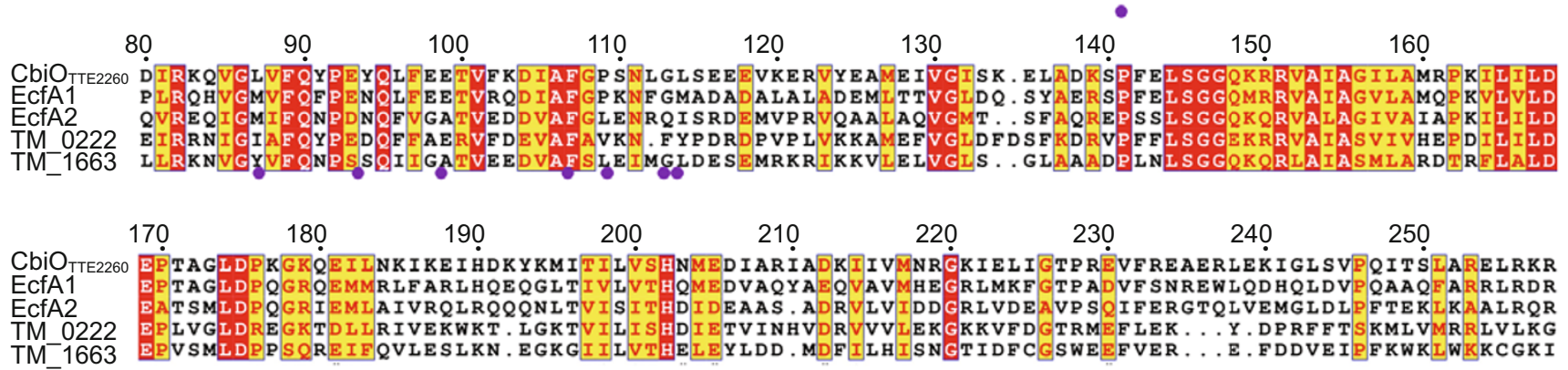

B

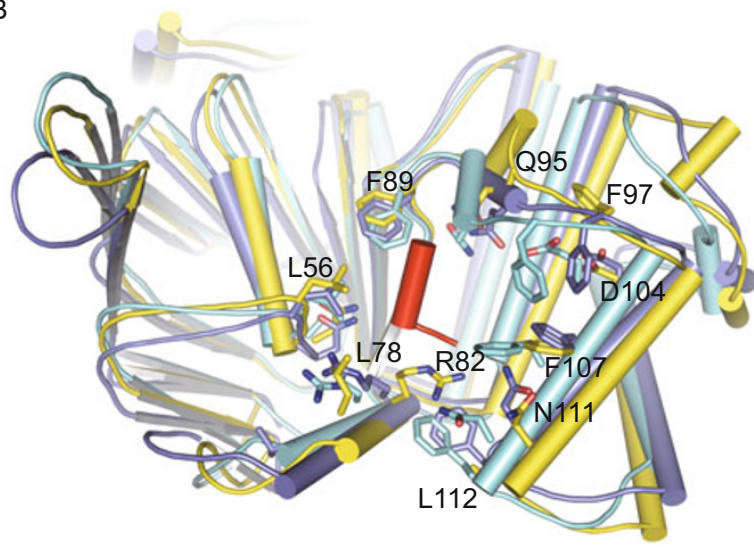

Figure 4. Arg82 from $\mathrm{CbiO}_{\text {TTE2260 }}$ adopts a unique rotamer. Top: sequence alignment of different $A$ components of ECF transporters. Identical and similar residues are highlighted with red and yellow grounds, respectively. The T component-interacting residues are indicated by solid circles at bottom. Bottom: structural alignment of $\mathrm{CbiO}_{\text {TTE2260 }}$ (light green), EcfA1 (slate) from 4HZU and TM_0222 (cyan) from $4 \mathrm{HLU}$. The side chains of EcfA from $4 \mathrm{HZU}$ interacting with the T component are shown in pink and their equivalent in $\mathrm{CbiO}_{\text {TTE2260 }}$ in yellow. 

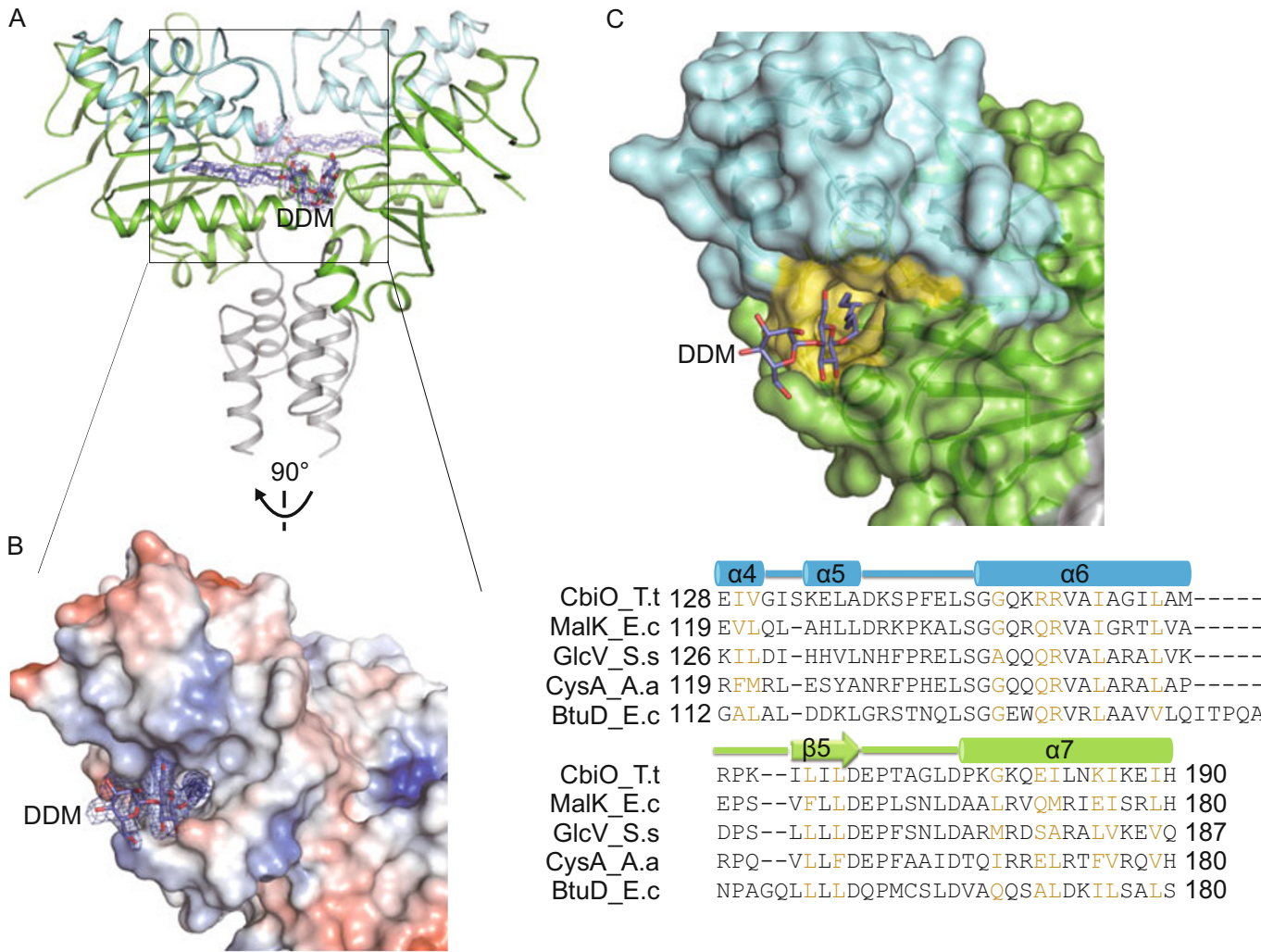

Figure 5. DDM binding sites and model. (A) Ribbon diagram (above) of $\mathrm{CbiO}_{\text {TTE2260 }}$ homodimer binding with two DDM molecules (represented in stick; $\mathrm{C}$ atom, slate; $\mathrm{O}$ atom, red.) and the electron density map of DDM is shown in blue mesh. (B) Close view and surface charge representation of $\mathrm{CbiO}_{\text {TTE2260 }}$ subunit molecule $\mathrm{A}$ binding with a DDM molecule (represented in stick; $\mathrm{C}$ atom, slate; $\mathrm{O}$ atom, red.). Positive charge is visualized in blue, negative charge is visualized in red while electrically neutral area is visualized in white. The electron density map of DDM is shown in blue mesh. (C) Surface and ribbon representation (above) of $\mathrm{CbiO}_{\text {TTE2260 }}$ molecule binding with DDM molecule shown in B. The lining of the DDM binding hydrophobic hole colored yellow, and the residues of DDM binding region from CbiO_T.t, MalK_E.c, ClcV_S.s, CysA_A.a and BtuD_E.c are aligned, and the residues constituted the lining of hydrophobic hole are visualized in dark yellow.

is substituted with the smaller residue Leu112 in $\mathrm{CbiO}_{\text {TTE2260 }}$ (Fig. 4A). Together, these subtle structural differences present Arg82 of $\mathrm{CbiO}_{\text {TTE2260 }}$ in a unique rotamer.

\section{Binding of DDM in $\mathrm{CbiO}_{\text {TTE2260 }}$ structure}

During opitimization of crystals, we found that addition of DDM significantly improved the resolution of $\mathrm{CbiO}_{\text {TTE2260 }}$ crystals. In the finally refined $\mathrm{CbiO}_{\text {TTE2260 }}$ structure, a DDM molecule well defined by electron density was found to bind to each of the two $\mathrm{CbiO}_{\text {TTE2260 }}$ (Fig 5A). The DDM hydrophobic tail inserts a hydrophobic hole of $\mathrm{CbiO}_{\text {TTE2260 }}$ (Fig 5B). Lining the hydrophobic hole are cluster of conserved hydrophobic residues, including lle129 and Vel130 from a4-helix, Gly147, Arg150, Arg151, Ile154 and Leu from a6-helix, Leu165 and Leu167 from B5strand and Gly178, Glu181, lle182, Lys185, lle186 and Ile189 from $\alpha 7$-helix (Fig $5 \mathrm{C}$ ). In addition to hydrophobic packing of the sugar rings against the main chain of a loop linking $\alpha 1$ and $\beta 2$, the hydrophilic head of DDM forms a number of hydrogen bonds with residues from the other $\mathrm{CbiO}_{\text {TTE2260. }}$. Thus, bind- ing of DDM can contribute to stabilizing the dimerization of $\mathrm{CbiO}_{\text {TTE2260. }}$. However, dimerization of $\mathrm{CbiO}_{\text {TTE2260 }}$ was DDMindependent (data not shown).

\section{DISCUSSION}

The CbiMNQO system is a founding member of the new class of ECF transporters (Rodionov et al., 2006; Siche et al., 2010). However, the stoichiometry of the transporter components remains unknown. The fact that a single A component (ATPase) encoded by the nik, cbi, and bio gene cassettes suggests that they may function as a homodimer. In the current study, we provide structural and biochemical evidence that the A component $\mathrm{CbiO}_{\text {TTE2260 }}$ in the CbiMNQO system exists as homodimers in crystals and solution. In the structure, a DDM molecule was found to bind to a conserved hydrophobic hole in the NBD domain. The bound DDM has a role in stabilizing the conformation of the NDB domain. But the biological significance remains further investigated. While homodimeric, the structural organization of the two $\mathrm{CbiO}$ molecules is strikingly similar to 
that of the heterodimeric dual A components found in other ECF transporters (Rodionov et al., 2006; Wang et al., 2013). In contrast with typical $A B C$ transporters, dimerization of the homo- or hetero-dimeric the A components in ECF is mainly mediated by the $\mathrm{C}$-terminal helical domain, forming a four-helix bundle. The conserved amino acids involved in formation of the helical bundle suggest that they are important for dimerization of $\mathrm{CbiO}$, either homo- or hetero-dimerization.

Structural studies define a 1:1:1:1 stoichiometry for the subgroup I ECF transporters containing dual A components (ter Beek et al., 2011). The 1:1:1 ratio in the ECF module is achieved through binding of two similar but not identical a-helices harboring the ARG motif from the T unit to two homologous $A$ units. An equal stoichiometry of this type of ECF transporter is further supported by light scattering studies of ECF transporters purified from L. lactis (Xu et al., 2013). However, a more recent biochemical study (Karpowich and Wang, 2013) suggested that a stoichiometry of $1: 1: 2: 2$ complex may exist for a similar ECF transporter, though further structural evidence for this is required. Although our current data do not allow us to determine the stoichiometry of the ECF module in the CbiMNQO system, the homodimeric $\mathrm{CbiO}_{\text {TTE2260 }}$ may suggest formation of a 2:2 ECF module. This hypothesis agrees with a recent study demonstrating existence of homo-dimeric T components in the BioMNY system (Neubauer et al., 2011). Moreover, in vivo FRET experiments using fluorescently labeled ECF transporters supported the presence of homo-oligomerized S component in each complex (Finkenwirth et al., 2010; Kirsch et al., 2012). It is important to note that how the CbiN component is involved in the assembly ECF transporters in the CbiMNQO system remains completely unknown. We cannot rule out the possibility that this component plays a role in the assembly of an ECF module. Clearly, further structural information is required to define the stoichiometry of the ECF transporters with a single A component.

Many structural and biochemical data have demonstrated that a conserved groove of A components is employed to binding to the ARG motif from the coupling helix of $T$ components (Neubauer et al., 2009; Neubauer et al., 2011; Wang et al., 2013). Mutation of the two Arg residues from the motif completely inactivated the activity of subgroup II ECF transporters (Neubauer et al., 2009). In contrast, similar mutations were tolerated to a certain extent in the BioMNY system (Neubauer et al., 2011). These data indicate that assembling the ECF module has different sensitivity to mutations of the ARG motif in the $\mathrm{T}$ components. Structural comparison of $\mathrm{CbiO}_{\text {TTE2260 }}$ with the ECF modules of other ECF transporters indicates that Arg82 of $\mathrm{CbiO}_{\text {TTE2260 }}$ completely overlaps with the ARG motif from the T components of other ECF modules, whereas Leu78 of CbiOTTE226 is positioned to disallow the Arg to adopt a similar rotamer to that in EcfA. Thus, unless striking conformational changes occur to $\alpha 2$ of $\mathrm{CbiO}_{\mathrm{TTE} 2260}$, the $\mathrm{T}$ components in the CbiMNQO system might have to use different sequences for interaction with $\mathrm{CbiO}_{\text {TTE2260. }}$. In keeping with this possibility, some of the T components in CbiMNQO lack a well-defined
ARG motif. These results suggest that residues outside the conserved $\mathrm{T}$ component binding groove on an $\mathrm{A}$ unit may also contribute to their specific interaction with each other.

\section{MATERIALS AND METHODS}

\section{Materials}

Pfu ploymerse, T4 DNA ligase, Fsel, Ascl and their corresponding buffer solutions were purchased from New England Biolabs. The detergent $n$-dodecyl- $\beta$-D-maltopyranoside (DDM) was from Affymetrix; gel filtration calibration kits and chromatographic columns, including Ni Sepharose 6 Fast Flow and Superdex 200 16/300 were from GE Healthcare; crystal screening kits were from Hamptom Research.

\section{Cloning, expression and purification}

The $\mathrm{CbiO}_{\text {TTE2260 }}$ gene (Accession No. NC_003869, Gene ID 997113) from $T$. tengcongensis was amplified by standard PCR_using the genomic DNA as a template. Sequences for the forward and reverse primer were 5'-TTGGCCGGCCGATGCCGATAAAAGTAGAAAAT-3' (with a Fsel cleavage site) and 5'-TTGGCGCGCCAACATTTTTTGTCCCCCTGAG-3' (with an Ascl cleavage site), respectively. The expression construct was generated by cloning the digested PCR product into the reconstructive prokaryotic expression vector $\mathrm{pET} 21 \mathrm{~b}$ (with a C-terminal His-tag). The recombinant plasmid was then transformed into the E. Coli strain BL21 (DE3). Cells were grown in LB (LutiaBertani) medium at $37^{\circ} \mathrm{C}$ to approximately $\mathrm{OD}_{600} 1.2$ and then IPTG $\left(0.5 \mathrm{mmol} / \mathrm{L}\right.$ final concentration) was added to induce $\mathrm{CbiO}_{\text {TTE2260 }}$ expression. Cells were allowed to grow for $8 \mathrm{~h}$ at $23^{\circ} \mathrm{C}$. The bacterial cells were harvested by centrifugation at $4000 \mathrm{rpm}$ for $15 \mathrm{~min}$ at $4^{\circ} \mathrm{C}$. The cells pellets were resuspended in lysis buffer $(20 \mathrm{mmol} / \mathrm{L}$ Tris $\mathrm{pH} 8.0,500 \mathrm{mmol} / \mathrm{L} \mathrm{NaCl}, 20 \mathrm{mmol} / \mathrm{L}$ imidazole, $1 \mathrm{mmol} / \mathrm{L} \mathrm{PMSF}$ ) and homogenized by sonication on ice. Supernatant was collected after centrifugation of the lysate at $15,000 \mathrm{rpm}$ for $1 \mathrm{~h}$ at $4^{\circ} \mathrm{C}$ and loaded onto an Ni Sepharose 6 Fast Flow column pre-equilibrated with buffer $\mathrm{A}$ $(20 \mathrm{mmol} / \mathrm{L}$ Tris $\mathrm{pH} 8.0,500 \mathrm{mmol} / \mathrm{L} \mathrm{NaCl}, 20 \mathrm{mmol} / \mathrm{L}$ imidazole, $0.002 \%$ (w/v) DDM). The column was then washed with buffer $\mathrm{B}(20 \mathrm{mmol} / \mathrm{L}$ Tris $\mathrm{pH} 8.0,500 \mathrm{mmol} / \mathrm{L} \mathrm{NaCl}, 40 \mathrm{mmol} / \mathrm{L}$ imidazole, 0.002\% (w/v) DDM). The bound proteins were eluted with buffer $\mathrm{C}(20 \mathrm{mmol} / \mathrm{L}$ Tris $\mathrm{pH} 8.0$, $500 \mathrm{mmol} / \mathrm{L} \mathrm{NaCl}, 300 \mathrm{mmol} / \mathrm{L}$ imidazole, $0.002 \%$ (w/v) DDM). The fraction containing the target protein was further purified by gel filtration using a Superdex $20010 / 300$ column in a buffer containing $20 \mathrm{mmol} / \mathrm{L}$ Tris $\mathrm{pH} 8.0,200 \mathrm{mmol} / \mathrm{L} \mathrm{NaCl}, 2 \mathrm{mmol} / \mathrm{L}$ DTT and $0.002 \%$ (w/v) DDM. The purified protein was then analyzed by SDS-PAGE followed by Coomasie staining. All the protein purification steps were performed at $4^{\circ} \mathrm{C}$.

\section{Crystallization and data collection}

The purified protein with a concentration $\sim 3.0 \mathrm{mg} / \mathrm{mL}$, was centrifuged at $13,000 \mathrm{rpm}$ for $30 \mathrm{~min}$ at $4^{\circ} \mathrm{C}$ to remove precipitants prior to crystal screening trials. Initial screening was performed at $18^{\circ} \mathrm{C}$ in a 48 -well plate using the sitting-drop vapor-diffusion method and sparse-matrix screening kits from Hampton Research. Initial conditions were refined with variations of $\mathrm{pH}$, precipitants and protein concentrations using the hanging-drop vapor-diffusion method consists of $1 \mu \mathrm{L}$ protein solution and $1 \mu \mathrm{L}$ reservoir solution. X-ray diffraction sets were collected at the Shanghai Synchrotron Radiation Facility (SSRF; Shanghai, People's Republic of China) BL17U with a wavelength of $0.9792 \AA$ on an ADSC 
Quantum 315r detector. A crystal was mounted in a nylon-fibre loop, immersed in a new drop of the reservoir solution for 5-10 s, and flashcooled in a liquid-nitrogen stream at $100 \mathrm{~K}$. The oscillation range was $1^{\circ}$ per frame and the exposure time was $1 \mathrm{~s}$ per frame with distance of the crystal to the detector $300 \mathrm{~mm}$. All diffraction data were indexed, integrated and scaled using the HKL-2000 programs DENZO and SCALEPACK (Otwinowski and Minor, 1997).

\section{Structure determination}

The $\mathrm{CbiO}_{\text {TTE2260 }}$ crystal structure was determined by molecular replacement using ARP/WARP in CCP4 (Collaborative Computational Project, 1994) with the $\mathrm{N}$-terminal domain of an $\mathrm{ABC}$ transporter from Thermotoga maritima (PDB 2YZ2) as the search module. The C-terminal of $\mathrm{CbiO}_{\text {TTE2260 }}$ was built manually using Coot (Emsley and Cowtan, 2004) after refinement of the model from molecular replacement. Water molecules were placed automatically using ARP/WARP and further checked manually using Coot. The final structure was refined with PHENIX (Adams et al., 2002) and contained residues 1-283 of each $\mathrm{CbiO}_{\text {TTE2260 }}$ subunit. The structure figures were illustrated using PyMOL molecular-graphics program (http://www.pymol.org).

\section{ACCESSION CODES}

The atomic coordinates and structure factors of the $\mathrm{CbiO}_{\text {TTE2260 }}$ have been deposited in the Protein Data Bank under accession code 4MKI.

\section{ACKNOWLEDGMENTS}

We would like to thank the staff at the SSRF BL17U beamline for assistance in data collection. This work was supported by the National Basic Research Program (973 Program) (Nos. 2011CB910502 and 2012CB911101) and the National Natural Science Foundation of China (Grant Nos. 31030020 and 31170679).

\section{ABBREVIATIONS}

CbiO, cobalt transporter ATP-binding subunit; DDM, n-dodecyl- $\beta$-Dmaltopyranoside; r.m.s.d., root mean square deviation

\section{COMPLIANCE WITH ETHICS GUIDELINES}

Chengliang Chai, You Yu, Wei Zhuo, Haifeng Zhao, Xiaolu Li, Na Wang, Jijie Chai and Maojun Yang declare that they have no conflict of interest.

This article does not contain any studies with human or animal subjects performed by the any of the authors.

\section{REFERENCES}

Adams, P.D., Grosse-Kunstleve, R.W., Hung, L.W., loerger, T.R., McCoy, A.J., Moriarty, N.W., Read, R.J., Sacchettini, J.C., Sauter, N.K., and Terwilliger, T.C. (2002). PHENIX: building new software for automated crystallographic structure determination. Acta Crystallogr D Biol Crystallogr 58, 1948-1954.

Berntsson, R.P., ter Beek, J., Majsnerowska, M., Duurkens, R.H., Puri, P., Poolman, B., and Slotboom, D.J. (2012). Structural divergence of paralogous $S$ components from ECF-type $A B C$ transporters. Proc Natl Acad Sci U S A 109, 13990-13995.

Chen, J., Lu, G., Lin, J., Davidson, A.L., and Quiocho, F.A. (2003). A tweezers-like motion of the ATP-binding cassette dimer in an ABC transport cycle. Mol Cell 12, 651-661.

Collaborative Computational Project, N. (1994). The CCP4 suite: programs for protein crystallography. Acta Crystallogr D Biol Crystallogr 50, 760-763.

Dean, M., and Allikmets, R. (1995). Evolution of ATP-binding cassette transporter genes. Curr Opin Genet Dev 5, 779-785.

Dean, M., Hamon, Y., and Chimini, G. (2001). The human ATP-binding cassette (ABC) transporter superfamily. J Lipid Res 42, 1007-1017.

Eitinger, T., Suhr, J., Moore, L., and Smith, J.A. (2005). Secondary transporters for nickel and cobalt ions: theme and variations. Biometals 18, 399-405.

Emsley, P., and Cowtan, K. (2004). Coot: model-building tools for molecular graphics. Acta Crystallogr D Biol Crystallogr 60, 2126-2132.

Erkens, G.B., Berntsson, R.P., Fulyani, F., Majsnerowska, M., VujicicZagar, A., Ter Beek, J., Poolman, B., and Slotboom, D.J. (2011). The structural basis of modularity in ECF-type ABC transporters. Nat Struct Mol Biol 18, 755-760.

Felsenstein, J. (1981). Evolutionary trees from DNA sequences: a maximum likelihood approach. J Mol Evol 17, 368-376.

Finkenwirth, F., Neubauer, O., Gunzenhauser, J., Schoknecht, J., Scolari, S., Stockl, M., Korte, T., Herrmann, A., and Eitinger, T. (2010). Subunit composition of an energy-coupling-factor-type biotin transporter analysed in living bacteria. Biochem $\mathrm{J} 431,373-380$.

Fisher, D.J., Fernandez, R.E., Adams, N.E., and Maurelli, A.T. (2012). Uptake of biotin by Chlamydia Spp. through the use of a bacterial transporter (BioY) and a host-cell transporter (SMVT). PLoS One 7, e46052.

Hung, L.W., Wang, I.X., Nikaido, K., Liu, P.Q., Ames, G.F., and Kim, S.H. (1998). Crystal structure of the ATP-binding subunit of an ABC transporter. Nature 396, 703-707.

Karpowich, N.K., and Wang, D.N. (2013). Assembly and mechanism of a group II ECF transporter. Proc Natl Acad Sci U S A 110, 25342539.

Kirsch, F., Frielingsdorf, S., Pohlmann, A., Ziomkowska, J., Herrmann, A., and Eitinger, T. (2012). Essential amino acid residues of BioY reveal that dimers are the functional $S$ unit of the Rhodobacter capsulatus biotin transporter. J Bacteriol 194, 4505-4512.

Korkhov, V.M., Mireku, S.A., and Locher, K.P. (2012). Structure of AMP-PNP-bound vitamin B12 transporter BtuCD-F. Nature 490, 367-372.

Li, X., Zhuo, W., Yu, J., Ge, J., Gu, J., Feng, Y., Yang, M., Wang, L., and Wang, N. (2013). Structure of the nucleotide-binding domain of a dipeptide $\mathrm{ABC}$ transporter reveals a novel iron-sulfur clusterbinding domain. Acta Crystallogr D Biol Crystallogr 69, 256-265.

Majsnerowska, M., Hanelt, I., Wunnicke, D., Schafer, L.V., Steinhoff, H.J., and Slotboom, D.J. (2013). Substrate-induced conformational changes in the S-component ThiT from an energy coupling factor transporter. Structure 21, 861-867.

Mulrooney, S.B., and Hausinger, R.P. (2003). Nickel uptake and utilization by microorganisms. FEMS Microbiol Rev 27, 239-261.

Neubauer, O., Alfandega, A., Schoknecht, J., Sternberg, U., Pohlmann, A., and Eitinger, T. (2009). Two essential arginine residues in the T components of energy-coupling factor transporters. J Bacteriol 191, 6482-6488.

Neubauer, O., Reiffler, C., Behrendt, L., and Eitinger, T. (2011). Interactions among the $\mathrm{A}$ and $\mathrm{T}$ units of an ECF-type biotin transporter 
analyzed by site-specific crosslinking. PLoS One 6, e29087.

Otwinowski, Z., and Minor, W. (1997). Processing of X-ray diffraction data collected in oscillation mode. Method Enzymol 276, 307-326.

Rodionov, D.A., Hebbeln, P., Gelfand, M.S., and Eitinger, T. (2006). Comparative and functional genomic analysis of prokaryotic nickel and cobalt uptake transporters: evidence for a novel group of ATPbinding cassette transporters. J Bacteriol 188, 317-327.

Scheffel, F., Demmer, U., Warkentin, E., Hulsmann, A., Schneider, E., and Ermler, U. (2005). Structure of the ATPase subunit CysA of the putative sulfate ATP-binding cassette $(A B C)$ transporter from Alicyclobacillus acidocaldarius. FEBS Lett 579, 2953-2958.

Siche, S., Neubauer, O., Hebbeln, P., and Eitinger, T. (2010). A bipartite $S$ unit of an ECF-type cobalt transporter. Res Microbiol 161, 824-829.

Story, R.M., Weber, I.T., and Steitz, T.A. (1992). The structure of the E. coli recA protein monomer and polymer. Nature 355, 318-325. ter Beek, J., Duurkens, R.H., Erkens, G.B., and Slotboom, D.J. (2011). Quaternary structure and functional unit of energy coupling factor (ECF)-type transporters. J Biol Chem 286, 5471-5475.

Verdon, G., Albers, S.V., Dijkstra, B.W., Driessen, A.J., and Thunnissen, A.M. (2003). Crystal structures of the ATPase subunit of the glucose $A B C$ transporter from Sulfolobus solfataricus: nucleotidefree and nucleotide-bound conformations. J Mol Biol 330, 343-358.

Wang, T., Fu, G., Pan, X., Wu, J., Gong, X., Wang, J., and Shi, Y. (2013). Structure of a bacterial energy-coupling factor transporter. Nature 497, 272-276.

Xu, K., Zhang, M., Zhao, Q., Yu, F., Guo, H., Wang, C., He, F., Ding, J., and Zhang, P. (2013). Crystal structure of a folate energy-coupling factor transporter from Lactobacillus brevis. Nature 497, 268-271.

Zhang, P., Wang, J., and Shi, Y. (2010). Structure and mechanism of the $S$ component of a bacterial ECF transporter. Nature 468, 717-720. 